\title{
Effects of wild type tau and disease-linked tau mutations on microtubule organization and intracellular trafficking
}

\author{
Dezhi Yu ${ }^{\mathrm{a}, \mathrm{b}}$, Stuart C. Feinstein ${ }^{\mathrm{b}, \mathrm{c}}$, Megan T. Valentine ${ }^{\mathrm{b}, \mathrm{d}}$ * \\ ${ }^{a}$ Department of Materials, University of California, Santa Barbara, Santa Barbara CA, USA \\ ${ }^{b}$ Neuroscience Research Institute, University of California, Santa Barbara, Santa Barbara CA, USA \\ ${ }^{c}$ Department of Molecular, Cellular and Developmental Biology, University of California, Santa Barbara, Santa \\ Barbara CA, USA \\ ${ }^{d}$ Department of Mechanical Engineering, University of California, Santa Barbara, Santa Barbara CA, USA \\ * Corresponding author: Department of Mechanical Engineering, Engineering II Building, Room 2361C, Santa \\ Barbara CA 93106, USA. valentine@engineering.ucsb.edu. (805)893-2594.
}

\begin{abstract}
We investigate the effects of transient expression of wild type (WT) and disease-linked mutations of tau (R406W, P301L, $\Delta \mathrm{N} 296)$ on cytoskeletal organization and cargo transport in COS-7 cells, which are natively tau-free. The introduction of tau proteins (either WT or mutant forms) leads to a dramatic restructuring of the microtubule cytoskeleton, as observed using immunofluorescence microscopy. Yet, this microtubule bundling and aggregation has a modest effect on the speed and travel distance of motor-driven cargo transport, as measured by the motions of fluorescently-labeled lysosomes. This suggests that localized transport events are insensitive to the global structure of the microtubule cytoskeleton. Importantly, we also found no evidence that the disease-linked tau mutants were particularly toxic; in fact we found that expression of mutant and WT tau had similar effects on overall microtubule structure and transport phenotypes.
\end{abstract}

Keywords: Microtubules, tau, intracellular transport

Submitted as an original article. 


\section{Introduction}

Tau proteins are expressed abundantly in human neurons, where they regulate microtubule dynamics, structure and organization (Dehmelt and Halpain, 2005; Panda et al., 1995). Tau mutations and misregulation can cause several closely related diseases including frontotemporal dementia with parkinsonism-17, progressive supranuclear palsy, Pick's disease and corticobasal degeneration, and aberrant tau action has long been associated with Alzheimer's pathology (Ballatore et al., 2007; Clark et al., 1998; Himmler, 1989; Himmler et al., 1989; Hong et al., 1998; Hutton et al., 1998; Kosik et al., 1989; Spillantini et al., 1998). Unfortunately, the molecular mechanisms by which these errors promote neurodegeneration and dementia remain poorly understood.

One often stated model hypothesizes that errors in cargo transport along microtubules may underlie some aspects of neurodegenerative diseases (Morfini et al., 2009; Sheng and Cai, 2012; Stokin et al., 2005), motivating numerous efforts to examine the role of tau in intracellular transport. In vitro measurements have shown that tau presence promotes motor dissociation; the effect is much larger for kinesin than dynein, leading to reduced anterograde transport efficiency (Dixit et al., 2008; Seitz et al., 2002; Vershinin et al., 2007). Cellular studies confirmed that wildtype tau reduces kinesin binding, leading to a redistribution of intracellular cargos, although the individual cargo speed is largely unaffected (Ebneth et al., 1998; Trinczek et al., 1999). However, few reports have focused specifically on the effects of disease-linked tau mutants on intracellular transport, so the link between the presence of pathological tau proteins and disruptions to intracellular trafficking remains largely unexplored. 
We previously showed that 4RS WT tau proteins and several disease-linked 4RS tau mutants (R406W, P301L, $\Delta \mathrm{N} 296)$ can reduce the speed of kinesin-driven cargo transport in vitro (Yu et al., 2013). Here, we extend that work to investigate the effects of transient expression of the same tau proteins on lysosome transport in COS-7 cells, which are natively tau-free. This allows us to explore the effects of tau mutations on microtubule organization and intracellular transport without the complication of a large endogenous tau population that could obscure the effects of the tau mutants. COS-7 and related cells are an excellent model for studies of intracellular transport: their flat geometry is ideal for fluorescence imaging, and they contain a well-organized microtubule cytoskeleton that spans the cell (Balint et al., 2013; Kapitein et al., 2010).

We found that introducing either WT or mutant tau proteins leads to a dramatic restructuring of the microtubule cytoskeleton, yet these changes have little effect on the speed or travel distance of motor-driven lysosome transport. This suggests that localized transport events are insensitive to the global structure of the microtubule cytoskeleton. Additionally, we found little evidence that the disease-linked tau mutants were particularly toxic. Rather, mutant and WT tau forms led to similar overall microtubule network structures and transport phenotypes. This suggests that tau mutations need not cause massive disruptions to microtubule organization or intracellular transport to effectively compromise or kill neuronal cells.

\section{Methods}

\subsection{Construction of tau expression vectors}


The PAAV-IRES-hrGFP (Strategene, 240075) expression vector containing a human cDNA insert for 4RS tau (4-repeat short: four microtubule binding domains, zero projection domain insertions, also called 4R0N) was used to express tau proteins in COS-7 cells. Tau expression is driven by a CMV promoter while GFP expression is driven by a separate internal ribosome entry site (IRES). Single point mutations (P301L, R406W and $\Delta$ N296) were introduced into the 4RS WT tau gene using the QuikChange II site-directed mutagenesis kit (Agilent Technologies, 200523).

\subsection{Cell culture and transfection}

COS-7 African green monkey kidney tissue cells were maintained at a subconfluent density in Dulbecco's Modified Eagle's Medium supplemented with 5\% horse serum (Cellgro, 35-030-CV), 5\% supplemented calf serum (HyClone, SH30072.03), $44 \mu \mathrm{M}$ sodium bicarbonate and $1 \%$ Antibiotic-Antimycotic (Gibco, \#15240-062) at $37^{\circ} \mathrm{C}$ and in a humidified, $7.5 \% \mathrm{CO}_{2}$ atmosphere. Cells were split 1:5 every $\sim 2-3$ days. For imaging, cells were trypsinized (Gibco, 25300-054), resuspended in antibiotic-free maintenance medium and plated on coverslipbottomed culture dishes (Bioptechs) coated with poly-L-lysine $(50 \mu \mathrm{g} / \mathrm{mL}$, Sigma) at a

concentration of $0.25 \times 10^{4}$ cells $/ \mathrm{cm}^{2}$ two hours prior to transfection. $1 \mu \mathrm{g}$ of DNA was transfected into the COS-7 cells 2-hrs after plating using PolyJet, per manufacturer's instructions (3 $\mu \mathrm{L}$, SignaGen, SL100688). The media was removed and replaced with fresh maintenance media 18-20 hours post-transfection. Fluorescence from the freely diffusing GFP allows rapid identification of transfected cells; transfection efficiency was typically $\sim 20 \%$.

\subsection{Fluorescence labeling, image acquisition, and lysosome tracking}


COS-7 cells were visualized using immunofluorescence microscopy 1-2 days after transfection. Thirty minutes prior to imaging, LysoTracker Deep Red reagent (50 nM, Life Technologies) was added to the cells at $37^{\circ} \mathrm{C}$ to fluorescently label the lysosomes. Media was replaced with Live Cell Image Solution (Life Technologies, A14291DJ) immediately before imaging. Cells were visualized with an Olympus DSU Confocal microscope, (100X oil immersion objective, $\mathrm{NA}=1.4$ ) with the spinning disk removed; a heated environmental enclosure maintained $37^{\circ} \mathrm{C}$. Images were obtained using a Hamamatsu ImaEM CCD camera; frame rate was $0.5 \mathrm{~Hz}$ for a collection time of 100 seconds. Lysosomes were tracked using the MOSAIC particle tracking algorithm available via ImageJ (Sbalzarini and Koumoutsakos, 2005). Tracking parameters were optimized as follows: Radius $=4$, Cutoff $=0$, Percentile $=0.1 \%$, Link Range $=2$, Displacement $=9$.

\subsection{Trajectory analysis}

To identify and isolate the motor-driven motions of the fluorescent lysosomes from the larger set of diffusive, non-directional motions we executed a series of selection steps. First, we eliminated all trajectories shorter than 30 frames. We then performed the Speed Correlation Index analysis, as described (Bouzigues and Dahan, 2007) to distinguish directed, processive motions ('runs') from those that are diffusing or are not moving at all (full details are provided in the Supplemental Materials). Segments with correlation indexes $>0.6$ that persisted for more than 15 points were selected for further analyses. A run was labeled anterograde (toward cell periphery) if the angle between the displacement vector and the radial vector from the cell center $<90^{\circ}$, all other cases were tagged retrograde (toward cell center). The mean speed and run length (total travel distance) of each run was also measured. 


\subsection{Immunofluorescence}

Cells were fixed by addition of $3.7 \%$ formaldehyde $\left(37^{\circ} \mathrm{C}\right.$, in water, 10 minutes $)$ then submerged in $100 \%$ methanol $\left(-20^{\circ} \mathrm{C}, 5\right.$ minutes $)$. Fixed cells were rehydrated with PBST buffer (0.1\% Triton-X-100 in PBS, 5 minutes, repeated for $3 \times$ total) incubated in blocking buffer $(1 \%$ BSA, 1\% donkey serum in PBST, 30 minutes), then incubated overnight with rabbit monoclonal anti- $\alpha$ tubulin antibody (1:500, EMD Milipore), then incubated overnight with Cy5-conjugated goat anti-rabbit secondary antibody (1:400, Jackson ImmunoResearch). All antibody incubations were done at room temperature. Cells were washed $4 \times, 8$ minutes/wash with PBST after each incubation. Images were obtained using Olympus Fluoview 1000 Spectral Confocal (60X silicone oil immersion objective, NA $=1.4)$.

\section{Results}

\subsection{The microtubule cytoskeleton is significantly reorganized upon the addition of tau}

To compare the effects of WT and disease-linked mutant isoforms of tau on microtubule network morphology in COS-7 cells, we used immunofluorescence to visualize the distribution of microtubule filaments 1-2 days post-transfection. We observed dramatic changes with several prominent features. (1) Instead of the long filamentous microtubules that we observed in cells without tau expression (Figure 1), we found bright punctate tubulin signals in cells that were transfected with tau after one day. These punctate spots indicate disruption of the normal filament structures and possibly aggregation of unpolymerized tubulins. (2) We observed thick microtubule bundles generally located near the cell peripheries in the majority of transfected 
cells (Figure 1), which transformed the microtubule network from the typical "aster-like" morphology of adherent cells to a circular, "wheel-like" geometry. (3) Lastly, we found the formation of microtubule bundles into extended projections between transfected cells and neighboring cells that are reminiscent of the microtubule laden projections observed in tau/baculovirus-expressing Sf9 cells (Figure 1) (Baas et al., 1991).

These phenotypic features were observed for all tau constructs: 4RS WT, 4RS P301L, 4RS R406W, and 4RS $\Delta \mathrm{N} 296$. These features appeared to be more prominent two days after transfection, when more bundling and more membrane association were observed (Figure 2). Additionally, increased overall tubulin fluorescence intensity was observed 2-days posttransfection as compared to cells that were transfected and then fixed after only one day. This suggests that tau addition promotes additional microtubule mass over time, perhaps as a result of the tubulin synthesis auto-regulatory mechanism, which responds to reduced tubulin subunit concentration by stabilizing tubulin mRNA, in turn leading to increased overall levels of tubulin (Gay et al., 1987).

\subsection{Impact of tau expression on bidirectional lysosome transport}

In contrast to our prior in vitro studies, the environment in the cellular system is very complex, and cellular cargoes are typically carried by multiple and different types of motors. As a result, cargoes in cells can move bidirectionally along microtubule filaments and exhibit qualitatively different motions, further complicated by geometric constraints and physical hindrances. To allow for quantitative measurements in this complex environment, we observed the directed motions of fluorescently-labeled lysosomes in COS-7 cells (see Methods). Directed 
runs were categorized as anterograde or retrograde based on the direction of the particle motion with respect to the cell center.

As shown in Figure 3 and Table S1, the mean velocities for anterograde and retrograde lysosome transport are similar for most conditions. Statistical analysis was performed using a two-sample Kolmogorov-Smirnov test (implemented in MATLAB; Table S2), which is nonparametric and can be used to compare any two datasets, without assumptions of Gaussianity or about the means or standard deviations of the distributions. We found no statistically significant difference in the anterograde and retrograde motions in any of the conditions based on a $95 \%$ confidence level, except for the 4RS P301L tau construct one day after transfection; by Day 2, this difference disappeared. In the remaining analysis, we continue to separate the anterograde and retrograde motions to allow subtle differences in motility to be discerned.

\subsection{Isoform-specific effects of tau on lysosome transport}

In control cells lacking tau, lysosomes transported in the anterograde direction had an average velocity of $0.39 \pm 0.04 \mu \mathrm{m} / \mathrm{s}$ (see Figure 3 and Table $\mathrm{S} 1$ for full reporting of the effects of all tau isoforms on lysosome velocity). One day after transfection, the average anterograde velocities were measured as follows: $0.31 \pm 0.03 \mu \mathrm{m} / \mathrm{s}$ for $4 \mathrm{RS} \mathrm{WT}, 0.28 \pm 0.02 \mu \mathrm{m} / \mathrm{s}$ for $4 \mathrm{RS}$ $\mathrm{P} 301 \mathrm{~L}, 0.27 \pm 0.03 \mu \mathrm{m} / \mathrm{s}$ for $4 \mathrm{RS} \mathrm{R} 406 \mathrm{~W}$, and $0.35 \pm 0.05 \mu \mathrm{m} / \mathrm{s}$ for $4 \mathrm{RS} \Delta \mathrm{N} 296$. When we compare the anterograde velocities of lysosomes moving in cells expressing any of the 4RS tau proteins (both WT and mutant isoforms) using a two-sample Kolmogorov-Smirnov test, we found a significant difference as compared to the speeds of cargos moving in the no-tau control cells. Here, and throughout, we assess significance for a $p$-value $<0.05 ; p$ values are listed in Tables S2 and S3. Additionally, the speed distribution of lysosomes moving in cells expressing 
any of the three of the $4 \mathrm{RS}$ mutants was significantly different from that of cargos moving in cells expressing 4RS WT tau. The mean lysosome speeds for cells expressing 4RS P301L and 4RS R406W were smaller than those of the 4RS WT expressing cells by $9.4 \%$ and $13.2 \%$, respectively. By contrast, the mean lysosome speed in cells expressing 4RS $\Delta \mathrm{N} 296$ increased slightly with respect to that of cells expressing 4RS WT tau, although the value was still reduced with respect to that of cargos moving in the no-tau control cells.

Two days after transfection, we measured the mean anterograde velocities as follows: $0.31 \pm 0.03 \mu \mathrm{m} / \mathrm{s}$ for $4 \mathrm{RS} \mathrm{WT}, 0.27 \pm 0.05 \mu \mathrm{m} / \mathrm{s}$ for $4 \mathrm{RS}$ P301L, $0.32 \pm 0.03 \mu \mathrm{m} / \mathrm{s}$ for $4 \mathrm{RS}$ $\mathrm{R} 406 \mathrm{~W}$, and $0.34 \pm 0.02 \mu \mathrm{m} / \mathrm{s}$ for $4 \mathrm{RS} \Delta \mathrm{N} 296$. The anterograde speed distribution for cells expressing 4RS WT constructs is significantly different than that of the no-tau control, with no change in mean speed between Day 1 and Day 2. When the lysosome speed distribution for cells expressing the mutant tau proteins is compared to that of the no-tau control cell on Day 2, only the 4RS R406W shows a significant difference, although the mean speed value for the 4RS R406W-expressing cells actually increases from Day 1 to Day 2. The $p$-value also increases from 0.01 to 0.045 , at the edge of our cutoff for significance. For the 4RS P301L- and 4RS $\Delta$ N296expressing cells, the mean speeds measured on Day 1 and Day 2 agree within the experimental uncertainty (estimated by the standard error of the mean (SEM)), and the distributions are indistinguishable from the no-tau control. Additionally, none of the speed distributions for cells expressing the 4RS mutant tau proteins differs significantly from that of the cells expressing 4RS WT on Day 2. This contrasts with the Day 1 results, in which all speed distributions for tau mutant-expressing cells showed a significant difference as compared to those expressing WT tau. Full statistical analysis is shown in Table S3. 
In retrograde transport, the average velocities dropped one day after transfection from $0.39 \pm 0.04 \mu \mathrm{m} / \mathrm{s}$ for control cells without tau, to $0.25 \pm 0.04 \mu \mathrm{m} / \mathrm{s}$ for cells expressing 4RS WT, $0.24 \pm 0.04 \mu \mathrm{m} / \mathrm{s}$ for $4 \mathrm{RS}$ P301L, $0.25 \pm 0.04 \mu \mathrm{m} / \mathrm{s}$ for $4 \mathrm{RS} \mathrm{R} 406 \mathrm{~W}$, and $0.34 \pm 0.04 \mu \mathrm{m} / \mathrm{s}$ for 4RS $\Delta$ N296 (Figure 3 and Table S1). The mean values of cargo transport speeds in the mutant tau-expressing cells are similar in anterograde and retrograde directions, whereas the mean speed of the retrograde motions in the 4RS WT-expressing are significantly reduced by $\sim 18 \%$ (the overall distributions are not distinguishable, see Table S2).

In our statistical analysis of cargo transport 1 day after transfection, we find a significant difference in the retrograde speed distributions of lysosomes moving in cells expressing any of the tau proteins as compared to the speeds of cargos moving in the no-tau control cells, in agreement with the results of our analysis of anterograde cargo motion. However, we do not measure any significant differences in the speed distribution of lysosomes moving in cells expressing any of the three of the 4RS mutants as compared to cells expressing 4RS WT tau. This differs from the case of anterograde transport, where significant differences were observed for all three mutants 1 day after transfection.

Two days after transfection, the mean lysosome velocities were measured to be $0.39 \pm$ $0.04 \mu \mathrm{m} / \mathrm{s}$ for the no-tau control cells, $0.24 \pm 0.04 \mu \mathrm{m} / \mathrm{s}$ for 4RS WT, $0.26 \pm 0.04 \mu \mathrm{m} / \mathrm{s}$ for $4 \mathrm{RS}$ P301L, $0.31 \pm 0.04 \mu \mathrm{m} / \mathrm{s}$ for 4RS R406W, and $0.37 \pm 0.04 \mu \mathrm{m} / \mathrm{s}$ for $4 \mathrm{RS} \Delta \mathrm{N} 296$. When we perform a statistical analysis, by comparing the retrograde velocity distribution of lysosomes moving in cells expressing 4RS WT to a no-tau control, we again find a significant reduction in speed on Day 2. However, none of speed distributions for cells expressing mutant forms of tau showed statistical difference as compared to the cargo speeds for cells without tau.

Interestingly, and in contrast to the anterograde cargo motions, we do observe significant 
differences in all three mutants when the speed distribution is compared to that of the 4RS WTexpressing cells on Day 2. However, this is not due to an increased disruption of cargo transport, rather the mean lysosome speed actually increases on Day 2 for each mutant form of tau. This leads to a greater difference with the speed data obtained from 4RS WT tau-expressing cells, which remain slower on the second day, and a closer agreement with the cargo speeds obtained using the no-tau control (see Table S3 for full analysis).

To further investigate the effects of WT and mutant tau proteins on lysosome motility, we also examined the distributions of lysosome velocities and run lengths for each condition (Figures S1 and S2). We found no obvious trends or outliers in the distribution histograms.

\section{Discussion}

We find that the expression WT and mutant tau proteins in COS-7 cells lacking endogenous tau causes reorganization of the microtubule cytoskeleton and impacts intracellular transport. Microtubule bundling increases in agreement with previous studies that investigated the effects of WT tau expressed in non-neuronal Sf9, COS and L cells (Baas et al., 1991; Kanai et al., 1992; Kanai et al., 1989; Takemura et al., 1992). We further observe tubulin aggregation, the formation of large bundled "wheels" of microtubules, and the presence of membraneassociated processes reminiscent of neuronal structures. Importantly, we do not observe any particular increase in toxicity upon expression of tau proteins harboring diseased-linked mutations, nor do we observe mutant-specific changes to cell morphology or cytoskeletal architecture. 
Next we investigated the effects of WT and mutant tau on intracellular transport. Despite the marked reorganization of the microtubule cytoskeleton, lysosome trafficking in tauexpressing cells was not dramatically different than in control cells lacking tau. This was particularly evident on the second day after transfection, when the disruption to the cytoskeletal structures became more prominent, but the impact on lysosome transport was either similar to that observed on Day 1 or was reduced. This suggests that localized transport events are insensitive to the global structure of the microtubule cytoskeleton, and that microtubule bundling and aggregation do not substantially impair motor-driven transport.

In all cases, the addition of tau reduced the mean cargo speed and significantly changed the cargo speed distributions compared to cargo motions in no-tau control cells in both the anterograde and retrograde directions. However, the mean velocities in the anterograde and retrograde directions were generally similar. In cells with a typical aster-like distribution of polarized microtubules, anterograde transport is powered primarily by kinesin-related motors, whereas retrograde transport is handled by dynein (Brown et al., 2005; Hirokawa et al., 1998). These two motor families have very different structures and stepping behaviors (Porter and Johnson, 1989; Woehlke and Schliwa, 2000), giving rise to differences in the velocities and regulation of retrograde and anterograde motion (Dixit et al., 2008; Hirokawa and Noda, 2008; Hirokawa et al., 1998).

There are several possible explanations for this observed lack of significant difference for for anterograde and retrograde transport in our study. In vivo bidirectional cargo transport is often described using a tug-of-war model in which kinesin and dynein motors compete (Gross et al., 2002; Hendricks et al., 2010; Kural et al., 2005; Muller et al., 2008). It is possible that the differential effects of tau on individual motors were masked by tug-of-war interactions on the 
multi-motor-bound lysosomes. It is also possible that the "wheel-like" microtubule networks we often observed confounded our assignment of motor direction as compared to those of the "asterlike" networks of non-transfected cells. The expression of tau may also promote spontaneous microtubule nucleation without centrosomes, which would disrupt the normal polarity of microtubules found in control cells (Witman et al., 1976).

In examining the effects of various point mutations in tau, we found the strongest reductions in cargo speed with the $\mathrm{P} 301 \mathrm{~L}$ mutation, which also had the strongest effect on kinesin translocation speed in vitro (Yu et al., 2013). The R406W mutant-expressing cells show similar effects to the P301L-expressing cells 1 day after transfection, but these effects are diminished on the second day. We find that the 4RS $\Delta$ N296 mutation has the most modest effect, with a small reduction in mean cargo speed with respect to the no-tau control cells, but an increase in cargo speed with respect to the 4RS WT tau-expressing cells on both Day 1 and 2, and in both the anterograde and retrograde directions.

In many cases, we observed an increase in the cargo speeds in the mutant tau-expressing cells on the second day after transfection. The molecular mechanism of this apparent recovery is not understood, but we can offer several possibilities. The tau mutants may lose their ability to regulate the intracellular transport system over time, allowing the endogenous COS-7 regulatory mechanisms to restore control. This would be consistent with our observation of higher tubulin/microtubule content in the tau-transfected cells on the second day. The observed recovery appears to be more prominent in the retrograde (dynein-driven) direction, which has been shown to be less sensitive to the presence of microtubule-associated proteins (Dixit et al., 2008). We also consider the possibility that our experimental design could influence our interpretation of results. For example, the apparent recovery could be influenced by selection 
bias, if cells with relatively high tau expression are so compromised two days post-transfection such that the only cells that were selected for measurement were those that were either more tolerant to the effects of tau, or those that had a lower overall expression of tau.

We also consider the possibility that the tau mutant proteins could impact transport mechanisms on time- or length-scales that are not currently accessible in our experimental system. Our imaging system allows us to observe all or nearly all of the cell volume in a single experiment, so it is unlikely that we are missing important parts of the trajectory due to lysosomes entering or leaving the observation volume. In support of this, the measured run length distributions monotonically decrease and with an exponential decay typical of motordriven transport. In terms of time scales, in order to reduce the effects of photobleaching, and to allow a reasonably large number of cells to be observed at each condition, we limit our total collection time for each cell to 100 seconds. We do not believe that this biases our motility data, as the majority of directed runs naturally end well before this time ( $\sim 5$ seconds is typical). We additionally require a minimum number of frames to be collected in order to distinguish directional motion and diffusion; in practice we eliminate all trajectories shorter than 30 frames (15 seconds). While it is possible that very short motor-driven events could exhibit different behaviors, including shorter trajectories would increase the uncertainty in determination of cargo speed and would increase the likelihood of including non-motor-driven diffusive events, so this was not explored.

The lack of mutant-specific changes in microtubule organization and intracellular transport is unexpected, and raises important questions about the role such mutations play in neurodegenerative diseases. The overall distribution of cargos within the cell is disrupted due to the changes in microtubule orientation and network morphology. These changes could 
cumulatively impact upon delicate intracellular regulatory mechanisms, leading to detrimental effects to cell activity and even viability over long times, perhaps in a manner that is dependent on the presence of point mutations in tau. Alternatively, it is well established that cells are incredibly sensitive to even subtle changes in microtubule dynamics, which tau proteins act to maintain within a narrow acceptable range (Bunker et al., 2006; Feinstein and Wilson, 2005; LeBoeuf et al., 2008). Perhaps the disease-linked mutations in tau act to disrupt this normal, but precarious, balance. Over time, this could impact upon axonal transport and/or other microtubule dependent activities. Perhaps future studies employing new advances in imaging technologies will allow simultaneous and high-resolution live-cell imaging of tau, kinesin, and dynein proteins as they act on dynamically growing and shrinking microtubules in order to resolve these open questions of the mechanisms of action in the tauopathies.

\section{Acknowledgements}

The authors thank Sarah Benbow, Nichole LaPointe, and Mary Raven for valuable discussions and technical advice. Authors gratefully acknowledge support from the National Science Foundation (CMMI-1254893 CAREER to MTV). We acknowledge the use of the NRI-MCDB Microscopy Facility and the Spectral Laser Scanning Confocal supported by the Office of The Director, National Institutes of Health of the NIH under Award \# S10OD010610. 


\section{Figure Captions}

Figure 1: Representative immunofluorescence images of control and tau-transfected COS-7 cells. The addition of tau caused disruption of the microtubule network. Scale bar is $30 \mu \mathrm{m}$. (A) Control cells exhibit normal organization of the microtubule cytoskeleton with few aggregates. (B) Cells expressing 4RS WT tau, one day after transfection, appear to have bright, punctate spots that may indicate unpolymerized tubulin aggregation (arrows). (C) Cells expressing 4RS R406W, two days after transfection show formations of thick microtubule bundles near cell peripheries (arrows). (D) Cells expressing 4RS $\Delta$ N296, one day after transfection, exhibit formation of localized microtubule bundles into projections that extend normal to the cell membrane (arrows).

Figure 2: Comparison of microtubule network morphology for cells that are (A) one day or (B) two days post-transfection with tau. Here, we show a cell expressing 4RS P301L, although this image is a representative of all tau proteins that were studied. After the second day, we observe more prominent bundling of microtubules near cell periphery and more tubulin/microtubule content, and in some cases the overall size of the cell appears to increase after expression of tau. Scale bar is $30 \mu \mathrm{m}$.

Figure 3: Mean velocities of lysosomes actively transported in the anterograde (black) or retrograde (gray) directions $(N=28-106)$. Error bars are SEM. 


\section{References}

Baas, P.W., Pienkowski, T.P., Kosik, K.S., 1991. Processes induced by tau expression in Sf9 cells have an axon-like microtubule organization. J. Cell Biol. 115, 1333-1344.

Balint, S., Verdeny Vilanova, I., Sandoval Alvarez, A., Lakadamyali, M., 2013. Correlative livecell and superresolution microscopy reveals cargo transport dynamics at microtubule intersections. Proceedings of the National Academy of Sciences of the United States of America 110, 3375-3380.

Ballatore, C., Lee, V.M., Trojanowski, J.Q., 2007. Tau-mediated neurodegeneration in Alzheimer's disease and related disorders. Nat. Rev. Neurosci. 8, 663-672.

Bouzigues, C., Dahan, M., 2007. Transient directed motions of GABA(A) receptors in growth cones detected by a speed correlation index. Biophysical Journal 92, 654-660.

Brown, C.L., Maier, K.C., Stauber, T., Ginkel, L.M., Wordeman, L., Vernos, I., Schroer, T.A., 2005. Kinesin-2 is a motor for late endosomes and lysosomes. Traffic 6, 1114-1124.

Bunker, J.M., Kamath, K., Wilson, L., Jordan, M.A., Feinstein, S.C., 2006. FTDP-17 mutations compromise the ability of tau to regulate microtubule dynamics in cells. The Journal of Biological Chemistry 281, 11856-11863.

Clark, L.N., Poorkaj, P., Wszolek, Z., Geschwind, D.H., Nasreddine, Z.S., Miller, B., Li, D., Payami, H., Awert, F., Markopoulou, K., 1998. Pathogenic implications of mutations in the tau gene in pallido-ponto-nigral degeneration and related neurodegenerative disorders linked to chromosome 17. Proceedings of the National Academy of Sciences of the United States of America 95, 13103-13107.

Dehmelt, L., Halpain, S., 2005. The MAP2/Tau family of microtubule-associated proteins. Genome Biol. 6, 204.

Dixit, R., Ross, J.L., Goldman, Y.E., Holzbaur, E.L., 2008. Differential regulation of dynein and kinesin motor proteins by tau. Science 319, 1086-1089.

Ebneth, A., Godemann, R., Stamer, K., Illenberger, S., Trinczek, B., Mandelkow, E., 1998. Overexpression of tau protein inhibits kinesin-dependent trafficking of vesicles, mitochondria, and endoplasmic reticulum: implications for Alzheimer's disease. J. Cell Biol. $143,777-794$.

Feinstein, S.C., Wilson, L., 2005. Inability of tau to properly regulate neuronal microtubule dynamics: a loss-of-function mechanism by which tau might mediate neuronal cell death. Biochim. Biophys. Acta 1739, 268-279.

Gay, D.A., Yen, T.J., Lau, J.T., Cleveland, D.W., 1987. Sequences that confer $\beta$-tubulin autoregulation through modulated mRNA stability reside within exon 1 of a $\beta$-tubulin mRNA. Cell 50, 671-679.

Gross, S.P., Welte, M.A., Block, S.M., Wieschaus, E.F., 2002. Coordination of opposite-polarity microtubule motors. J. Cell Biol. 156, 715-724.

Hendricks, A.G., Perlson, E., Ross, J.L., Schroeder, H.W., 3rd, Tokito, M., Holzbaur, E.L., 2010. Motor coordination via a tug-of-war mechanism drives bidirectional vesicle transport. Current biology : CB 20, 697-702.

Himmler, A., 1989. Structure of the bovine tau gene: alternatively spliced transcripts generate a protein family. Molecular and Cellular Biology 9, 1389-1396. 
Himmler, A., Drechsel, D., Kirschner, M.W., Martin, D., 1989. Tau consists of a set of proteins with repeated $\mathrm{C}$-terminal microtubule-binding domains and variable $\mathrm{N}$-terminal domains. Molecular and Cellular Biology 9, 1381-1388.

Hirokawa, N., Noda, Y., 2008. Intracellular transport and kinesin superfamily proteins, KIFs: structure, function, and dynamics. Physiol. Rev. 88, 1089-1118.

Hirokawa, N., Noda, Y., Okada, Y., 1998. Kinesin and dynein superfamily proteins in organelle transport and cell division. Curr. Opin. Cell. Biol. 10, 60-73.

Hong, M., Zhukareva, V., Vogelsberg-Ragaglia, V., Wszolek, Z., Reed, L., Miller, B.I., Geschwind, D.H., Bird, T.D., McKeel, D., Goate, A., 1998. Mutation-specific functional impairments in distinct tau isoforms of hereditary FTDP-17. Science 282, 1914-1917.

Hutton, M., Lendon, C.L., Rizzu, P., Baker, M., Froelich, S., Houlden, H., Pickering-Brown, S., Chakraverty, S., Isaacs, A., Grover, A., 1998. Association of missense and 5'-splice-site mutations in tau with the inherited dementia FTDP-17. Nature 393, 702-705.

Kanai, Y., Chen, J., Hirokawa, N., 1992. Microtubule bundling by tau proteins in vivo: analysis of functional domains. The EMBO Journal 11, 3953-3961.

Kanai, Y., Takemura, R., Oshima, T., Mori, H., Ihara, Y., Yanagisawa, M., Masaki, T., Hirokawa, N., 1989. Expression of multiple tau isoforms and microtubule bundle formation in fibroblasts transfected with a single tau cDNA. J. Cell Biol. 109, 1173-1184.

Kapitein, L.C., Schlager, M.A., Kuijpers, M., Wulf, P.S., van Spronsen, M., MacKintosh, F.C., Hoogenraad, C.C., 2010. Mixed microtubules steer dynein-driven cargo transport into dendrites. Current Biology 20, 290-299.

Kosik, K.S., Orecchio, L.D., Bakalis, S., Neve, R.L., 1989. Developmentally regulated expression of specific tau sequences. Neuron 2, 1389-1397.

Kural, C., Kim, H., Syed, S., Goshima, G., Gelfand, V.I., Selvin, P.R., 2005. Kinesin and dynein move a peroxisome in vivo: a tug-of-war or coordinated movement? Science 308, 14691472.

LeBoeuf, A.C., Levy, S.F., Gaylord, M., Bhattacharya, A., Singh, A.K., Jordan, M.A., Wilson, L., Feinstein, S.C., 2008. FTDP-17 mutations in tau alter the regulation of microtubule dynamics - an "Alternative Core" model for normal and pathological tau action. The Journal of Biological Chemistry 238, 36406-36415.

Morfini, G.A., Burns, M., Binder, L.I., Kanaan, N.M., LaPointe, N., Bosco, D.A., Brown, R.H., Brown, H., Tiwari, A., Hayward, L., 2009. Axonal transport defects in neurodegenerative diseases. The Journal of Neuroscience 29, 12776-12786.

Muller, M.J., Klumpp, S., Lipowsky, R., 2008. Tug-of-war as a cooperative mechanism for bidirectional cargo transport by molecular motors. Proceedings of the National Academy of Sciences of the United States of America 105, 4609-4614.

Panda, D., Goode, B.L., Feinstein, S.C., Wilson, L., 1995. Kinetic stabilization of microtubule dynamics at steady state by tau and microtubule-binding domains of tau. Biochemistry 34, 11117-11127.

Porter, M.E., Johnson, K.A., 1989. Dynein structure and function. Annu. Rev. Cell Biol. 5, 119151.

Sbalzarini, I.F., Koumoutsakos, P., 2005. Feature point tracking and trajectory analysis for video imaging in cell biology. Journal of Structural Biology 151, 182-195. 
Seitz, A., Kojima, H., Oiwa, K., Mandelkow, E.M., Song, Y.H., Mandelkow, E., 2002. Single-molecule investigation of the interference between kinesin, tau and MAP2c. The EMBO journal 21, 4896-4905.

Sheng, Z.-H., Cai, Q., 2012. Mitochondrial transport in neurons: impact on synaptic homeostasis and neurodegeneration. Nature Reviews Neuroscience 13, 77-93.

Spillantini, M.G., Murrell, J.R., Goedert, M., Farlow, M.R., Klug, A., Ghetti, B., 1998. Mutation in the tau gene in familial multiple system tauopathy with presenile dementia. Proceedings of the National Academy of Sciences of the United States of America 95, 7737-7741.

Stokin, G.B., Lillo, C., Falzone, T.L., Brusch, R.G., Rockenstein, E., Mount, S.L., Raman, R., Davies, P., Masliah, E., Williams, D.S., 2005. Axonopathy and transport deficits early in the pathogenesis of Alzheimer's disease. Science 307, 1282-1288.

Takemura, R., Okabe, S., Umeyama, T., Kanai, Y., Cowan, N.J., Hirokawa, N., 1992. Increased microtubule stability and alpha tubulin acetylation in cells transfected with microtubuleassociated proteins MAP1B, MAP2 or tau. Journal of Cell Science 103 ( Pt 4), 953-964.

Trinczek, B., Ebneth, A., Mandelkow, E., Mandelkow, E., 1999. Tau regulates the attachment/detachment but not the speed of motors in microtubule-dependent transport of single vesicles and organelles. Journal of Cell Science 112, 2355-2367.

Vershinin, M., Carter, B.C., Razafsky, D.S., King, S.J., Gross, S.P., 2007. Multiple-motor based transport and its regulation by Tau. Proceedings of the National Academy of Sciences of the United States of America 104, 87-92.

Witman, G.B., Cleveland, D.W., Weingarten, M.D., Kirschner, M.W., 1976. Tubulin requires tau for growth onto microtubule initiating sites. Proceedings of the National Academy of Sciences of the United States of America 73, 4070-4074.

Woehlke, G., Schliwa, M., 2000. Walking on two heads: the many talents of kinesin. Nature reviews. Molecular Cell Biology 1, 50-58.

Yu, D., LaPointe, N.E., Guzman, E., Pessino, V., Wilson, L., Feinstein, S.C., Valentine, M.T., 2014. Tau Proteins Harboring Neurodegeneration-Linked Mutations Impair Kinesin Translocation in vitro. Journal of Alzheimer's Disease 39, 301-314. 





